\title{
Lifetime of silica final lenses subject to HiPER irradiation conditions
}

\author{
A. Rivera ${ }^{\text {a }}$, D. Garoz ${ }^{\mathrm{a}}$, R. Juarez ${ }^{\mathrm{a}, \mathrm{b}}$, J. Alvarez ${ }^{\mathrm{a}}$, R. González-Arrabal ${ }^{\mathrm{a}}$, J.M. Perlado ${ }^{\mathrm{a}}$ \\ ${ }^{a}$ Instituto de Fusión Nuclear, UPM, Jose Gutierrez Abascal 2, E28006 Madrid, Spain; \\ ${ }^{\mathrm{b}}$ Departamento de ingeniería energética, UNED, Juan del Rosal 12, E28040 Madrid, Spain
}

\begin{abstract}
The goal of the European laser fusion project, is to build an engineering facility for repetitive laser operation (HiPER 4a) and later a fusion reactor (HiPER 4b). A key aspect for laser fusion energy is the final optics. At the moment, it is based on silica transmission lenses located $8 \mathrm{~m}$ away from the chamber center. Lens lifetime depends on the irradiation conditions. We have used a $48 \mathrm{MJ}$ shock ignition target for calculations. We have studied the thermo-mechanical effects of ions and X-rays on the lenses. Ions lead to lens melting and must therefore be mitigated. On the other hand, X-rays ( $\sim 1 \%$ of the energy) does not produce either a significant temperature rise or detrimental stresses. Finally, we calculated the neutron flux and gamma dose rate on the lenses. Next, based on a simple model we studied the formation of color centers in the sample, which lead to optical absorption. Calculations show that simultaneous neutron and gamma irradiation does not significantly increase the optical absorption during the expected lifetime of the HiPER 4a facility. Under severe conditions (HiPER 4b), operation above $800 \mathrm{~K}$ or lens refreshing by thermal annealing treatments seem to assure adequate behavior.
\end{abstract}

Keywords: Inertial Fusion, Final Optics, Neutron Irradiation, Materials Damage, Silica

\section{INTRODUCTION}

The European laser fusion project, HiPER (High Power laser Energy Research facility) is now in the final year of the preparatory phase (phase 2). Over the next 7 years, a technological phase (HiPER phase 3) will take place to minimize construction risks through appropriate $R \& D$ activities. The goal is to build an engineering facility for repetitive laser operation (HiPER 4a) working with bunches of 100 shots with up to 5 ignition shots and a maximum energy per bunch of about $100 \mathrm{MJ}$. Next, (circa 5 years later) a reactor to demonstrate commercial viability of laser fusion energy will be built (HiPER 4b). Thus, it will have to operate continuously at full power producing its own tritium and generating electric power. An overview of the operation scenarios for HiPER $4 \mathrm{a}$ and $4 \mathrm{~b}$ phases is presented in Table 1.

A very important issue for the development of laser fusion is the final optics. The different components involved must perform the frequency conversion, wavelength separation and focusing of each bundle in the beam line at a small spot on the target. Therefore, the ignition process itself depends on the final optics.

Despite efforts to minimize radiation threats, the final focusing element of each beam line must face the target. If needed, to preserve the properties of these elements, ions, electrons and X-rays can be (fully or partially) mitigated by means of different strategies. However, the neutron pulses derived from every explosion as well as the $\gamma$-radiation resulting from $(\mathrm{n}, \gamma)$ reactions and chamber activation are unavoidable.

HiPER proposes the use of transmission lenses for focusing, being silica the primary material of choice due to its cost, optical properties and radiation resistance ${ }^{1,2}$. Silica is a structurally-simple glassy material with band gap energy of $\sim 8$ $\mathrm{eV}$ that presents good optical transparency from around $300 \mathrm{~nm}$ to the visible band. Therefore, it covers the $2^{\text {nd }}$ and $3^{\text {rd }}$ harmonic wavelengths of a typical solid-state laser for fusion energy.

* antonio.rivera.mena@gmail.com, tel. +34 9133631 10, fax +34 913363002 
Table 1. Operation scenarios planned for HiPER construction phases $4 \mathrm{a}$ and $4 \mathrm{~b}$.

\begin{tabular}{l|l|l} 
& HiPER 4a & HiPER 4b \\
\hline Description & $\begin{array}{l}\text { Experimental } \\
\text { facility }\end{array}$ & $\begin{array}{l}\text { Demonstration } \\
\text { reactor } \\
\text { Operation }\end{array}$ \\
$\begin{array}{l}\text { Bunches of 100 } \\
\text { shots, max. 5 DT } \\
\text { explosions }\end{array}$ & \\
Continuous (24/7) \\
Yield (MJ) & $<20$ & $>100$ \\
Rep. rate (Hz) & $1-10$ & $10-20$ \\
Power (GWt) & - & $1-3$ \\
T cycle & No & Yes \\
Blanket & No & Yes
\end{tabular}

Point defects are produced in silica by means of particle irradiation and by purely ionizing radiation, such as $\gamma$-rays. These defects constitute color centers that turn out to be detrimental because they are the origin of unwanted laser absorption and scattering ${ }^{3}$. It is now well ascertained that irradiation of silica with fast neutrons induces two main effects: network compaction (densification) ${ }^{4}$ and point defect generation ${ }^{5,6}$. On the other hand, purely ionizing radiation only produces color centers ${ }^{7-9}$. The simultaneous irradiation with neutrons and $\gamma$-rays (as it is the case in a laser fusion reactor) leads to synergistic effects resulting in an enhanced degradation of the optical transmission properties ${ }^{2,10,11}$.

Defects in silica are very complex. They can be charged or uncharged and interact among themselves giving rise to different configurations depending on temperature, stoichiometry and irradiation conditions ${ }^{12,13}$. In addition, the presence of extrinsic defects, in particular hydrogen-related defects play a crucial role in defect configuration ${ }^{14}$. We will focus here only on the defects detected after simultaneous neutron and $\gamma$-ray irradiation. We will apply the simple model for defect evolution developed in Refs. 10 and 11 to the realistic conditions expected in laser fusion with low yield shockignition direct targets as those proposed for HiPER 4a. In brief, the model takes into account changes in optical absorption as a consequence of color center formation by radiation. Neutron irradiation leads to oxygen defect centers $(\mathrm{ODC})$, i.e., oxygen vacancies (absorption peak at $248 \mathrm{~nm}$ ) and non-bridging oxygen hole centers (NBOHC). ODC act as precursors of E' centers being the $\gamma$-radiation that converts ODC into E' centers (absorption peak at $214 \mathrm{~nm}$ ). The sample temperature plays a central role because defects are thermally annealed out restoring the original silica network.

The purpose of this work is to estimate the lifetime of the silica final lens proposed within the HiPER 4a scheme. Starting with the spectra obtained by LASNEX calculations for a 48 MJ shock ignition target ${ }^{15,16}$, we estimate realistic radiation fluxes in the silica lenses located at $8 \mathrm{~m}$ from the target. Next, we calculate the themomechanical response of the silica sample to the radiation. The conclusion is that ions must be mitigated due to fatal damage in the final silica lenses. On the other hand, calculations show that since X-rays deposit much less energy, the silica lenses will survive Xray radiation. Finally, the formation of color centers upon neutron and gamma irradiation and the subsequent enhancement in optical absorption is discussed in terms of the simple model of color center formation ${ }^{10,11}$. As an overall conclusion, our calculations indicate that the lifetime of the lenses is comparable to the lifetime of the HiPER 4a facility.

\section{HIPER FINAL OPTICS ASSEMBLY (FOA)}

Advanced designs exist for HiPER 4a. Figure 1 shows schematically a proposed design that has been extensively studied including aspects such as chamber protection, final optics protection, optical system design, target injection, radiation shielding and remote handling. According to this design, the reactor chamber consists of a $5 \mathrm{~m}$ inner radius spherical steel (T91 alloy) chamber with 48 laser beam lines for symmetrical illumination of direct targets. The chamber thickness is $10 \mathrm{~cm}$ and has a $1 \mathrm{~mm}$-thick $\mathrm{W}$ armor. The outer surface is surrounded by $80 \mathrm{~cm}$-thick borated concrete for shielding. The laser beams are transported to the chamber in steel (T91) tubes with a square section of $1 \times 1 \mathrm{~m}^{2}$ and $1 \mathrm{~cm}$-thick walls. The final lenses are made of fused silica and are located $8 \mathrm{~m}$ away from the chamber center. These lenses are squares of $75 \times 75 \mathrm{~cm}^{2}$ with a thickness of $5 \mathrm{~cm}$. At $19 \mathrm{~m}$ from the chamber center the remaining components of the final optics are located. They are shielded by a concrete wall located at $16 \mathrm{~m}$ from the chamber center. The wall has small 
openings (pinholes) for effective shielding. This configuration strongly decreases the neutron flux beyond the wall. Finally, a $2.5 \mathrm{~m}$-thick cylindrical bioshield surrounds the target bay. Note that the final focusing lenses directly face the target. This implies that these lenses are subject to severe irradiation conditions that presumably could make necessary to replace them periodically or use healing methods such as annealing to reestablish their optical properties. For this reason, they are sometimes called "renewable lenses", see Figure 1.

Other designs, for example $\mathrm{HAPL}^{17}$, propose the use of mirrors far away from the target. Such a scheme assures a minimum radiation load onto the mirrors at the expense of using long focal lengths. The scheme proposed for HiPER employs lenses with short focal lengths appropriate to achieve the small focal spots that are required for optimum illumination of the direct targets.

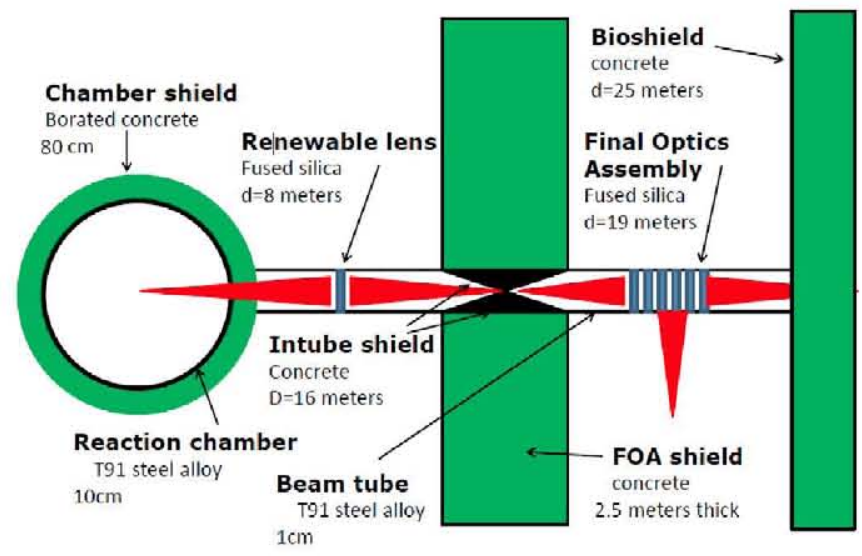

Figure 1. Schematic representation of the HiPER 4a facility (not on scale). Distances from the target (chamber center) are indicated. Three main elements are shown from left to right: (1) the steel chamber with a W armor for wall protection, surrounded by borated concrete for shielding (see the final lens at $8 \mathrm{~m}$ from the target); (2) the final optics shield with a pinhole to minimize neutron irradiation of final optics; (3) the biodshield for environment protection.

\section{TARGET YIELD}

The ignition scheme that HiPER will use is not decided yet. However, high gain ignition schemes, such as fast ignition and shock ignition, with direct drive targets are preferred and will be investigated in the HiPER 4a facility. In order to get conclusions on silica response to HiPER explosions, we used radiation spectra (Figure 2) obtained by LASNEX calculations for a $48 \mathrm{MJ}$ shock ignition target ${ }^{15,16}$. This target yield is an upper limit for HiPER $4 \mathrm{a}$, which according to Table 1 will employ targets of up to $20 \mathrm{MJ}$.

It is very relevant to note that for the $48 \mathrm{MJ}$ target, $75 \%$ of the energy is released as neutrons, $24 \%$ in the form of ions and the rest (1\%) in the form of X-rays. This is typical for direct drive targets. In the case of ions, we can distinguish burn product ions and debris ions. The former are mainly ${ }^{4} \mathrm{He}$ ions born in fusion reactions, which reach the final lens with a pulse width of a few hundred ns and constitute $\sim 8 \%$ of the target yield. The latter correspond to ions stemming from the hot plasma, mainly $\mathrm{D}$ and $\mathrm{T}$, which reach the final lens with a pulse width of a few $\mu$ s. Since ions deposit all their kinetic energy in the first few $\mu \mathrm{m}$ of the material in these short time scales, they contribute to a huge thermal load on the lenses with significant thermo-mechanical effects, as discussed in the next section.

In order to estimate the neutron and gamma fluxes received by the final lenses as a function of time and energy, we use the neutron spectrum shown in Figure 2 as source term for MCNPX ${ }^{18}$ calculations. The geometry described in the previous section is modeled with $\mathrm{MCAM}^{19}$ translated to MCNPX. Figure 3 shows the neutron flux obtained by means of these calculations. The inset contains the neutron energy reaching the final lenses as a function of time. MCNPX was also used to calculate the $\gamma$-dose absorbed by the silica final lenses. Figure 3 shows the $\gamma$-dose rate as a function of time. Note that a prompt $\gamma$-flux due to $(\mathrm{n}, \gamma)$ reactions accompanies the neutron peak, i.e., the final lenses are subject to simultaneous neutron and $\gamma$-radiation arriving as pulses of high flux. 


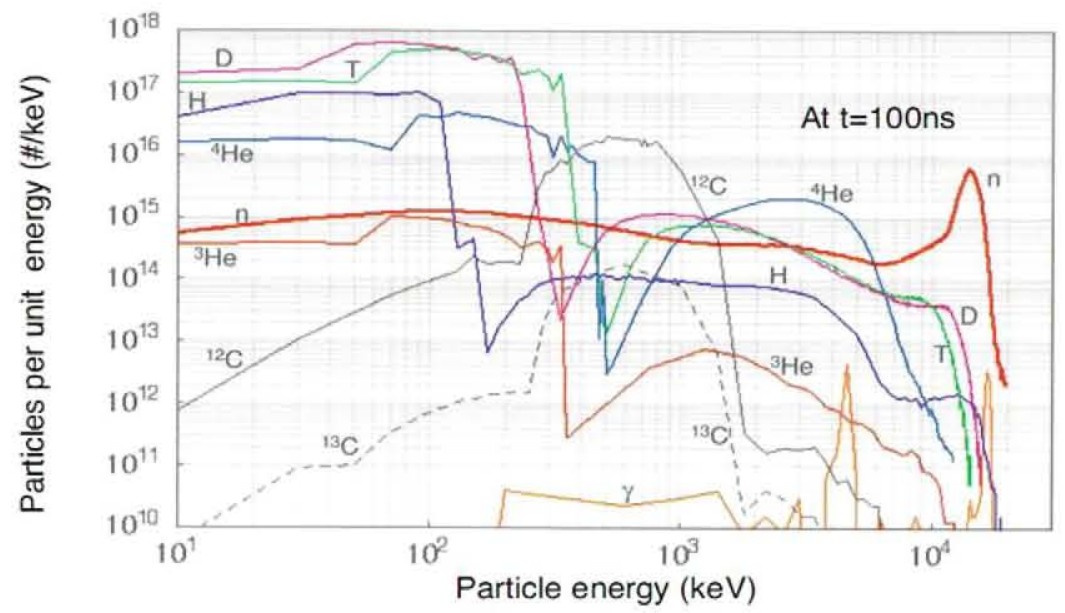

Figure 2. Radiation spectra obtained by means of LASNEX calculations for a 48 MJ shock ignition target.

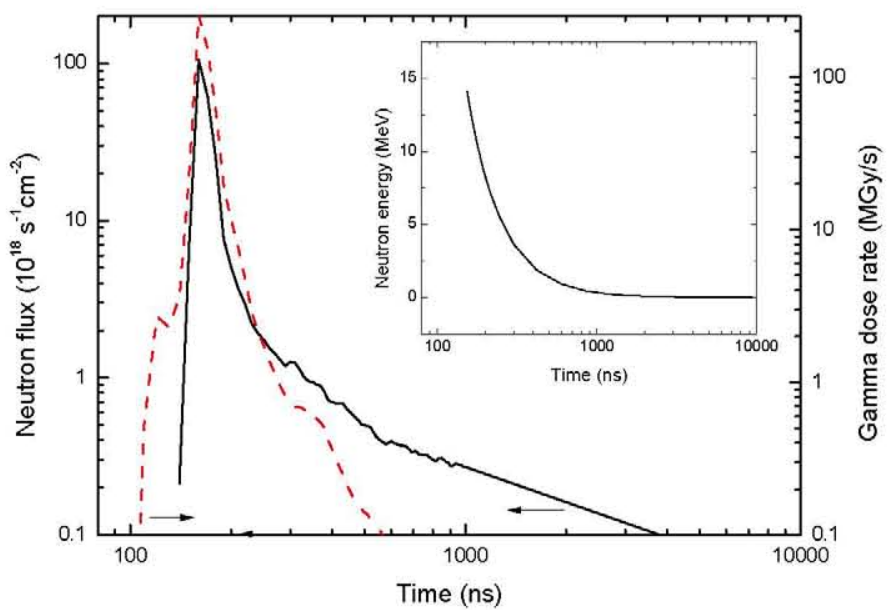

Figure 3. (Left axis) neutron flux, (right axis) gamma dose rate and (inset) neutron energy as a function of time after the explosion.

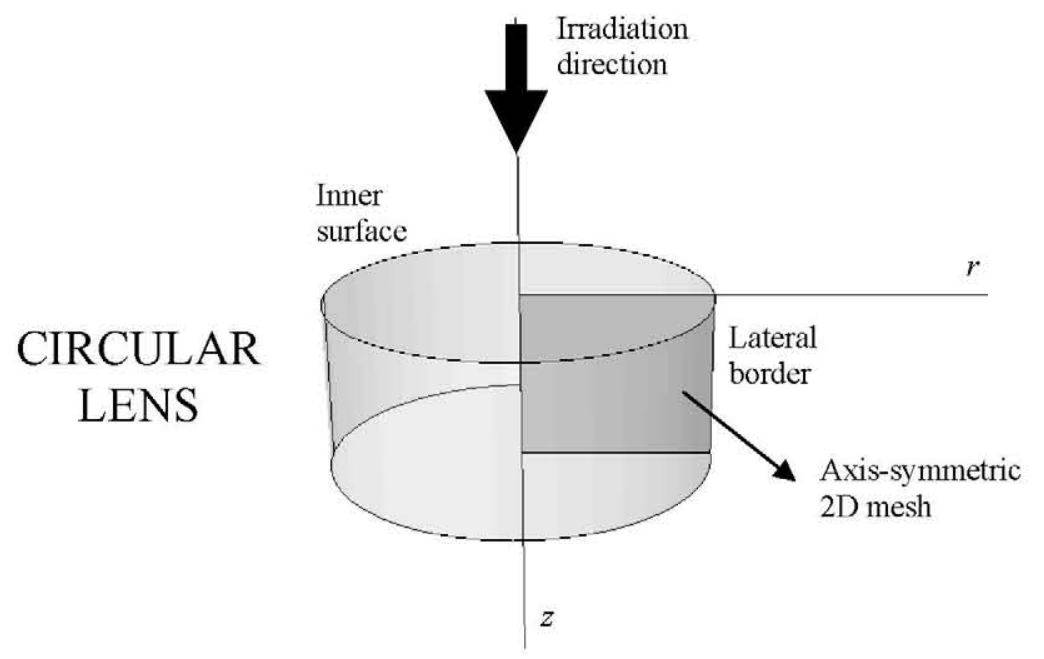

Figure 4. Schematic representation of a circular lens irradiated parallel to the lens axis $(z)$. For calculation purposes the lens is represented by a $2 \mathrm{D}$ axis-symmetric mesh. 


\section{THERMO-MECHANICAL RESPONSE}

In order to account for the themo-mechanical response of the silica lenses located at $8 \mathrm{~m}$ from the target, we carried out calculation by means of finite elements making use of the multi-physics open software CODE ASTER ${ }^{20}$. This software allows one to vary the physical and mechanical constants as a function of temperature. We have reduced the 3D problem to a 2D problem assuming circular lenses, which are axis-symmetric about their axis (see Figure 4). This improves significantly the necessary computation time. The calculations were carried out with a mesh formed by rectangular elements. The mesh is adapted to the problem in such a way that close to the inner surface, where most energy is deposited, the dimensions of the elements are of the order of $1 \mathrm{~nm}$. In deep regions not affected by radiation these dimensions go up to $1 \mathrm{~mm}$.

The final lenses are subject to a significant thermal load after every explosion in the form of ions and X-rays. The contribution of both neutrons and gamma-radiation is much lower because such penetrating forms of radiation deliver their energy over a large volume. From the ion spectra resulting from the $48 \mathrm{MJ}$ explosion shown in Figure 2 one can obtain the time-dependent flux on the final lenses. On the other hand, the energy depth distribution depends on the nature of the incoming radiation and its energy. We calculated the energy depth profile for ions by means of the code SRIM ${ }^{21}$. The range corresponding to ${ }^{4} \mathrm{He}$ with average energy of $2.1 \mathrm{MeV}$ is $6.4 \mu \mathrm{m}$, while that corresponding to $\mathrm{D}$ with average energy of $150 \mathrm{keV}$ is $1.4 \mu \mathrm{m}$. Therefore, ions carry $\sim 1 / 4$ of the target energy, which is deposited in a very shallow region of the sample. This fact leads to complete melting of the near surface region. The main conclusion is that ion irradiation has a deleterious effect on the sample. If we rescale the output energy of the target to $24 \mathrm{MJ}$ to approach the expected situation in HiPER ta we obtain similar conclusions.

Therefore, strategies to mitigate (at least partially) the ion radiation on the final lenses is absolutely necessary. Several strategies have been studied. Contrary to the case of indirect drive targets, with direct drive targets gas protection is a bad option. This is due to the fact that (i) the presence of a residual gas pressure (typically $100 \mathrm{~Pa}$ of $\mathrm{Xe}$ at standard temperature) makes difficult to keep the target temperature below its triple point ${ }^{22}$; (ii) direct target tracking is difficult with the presence of a residual gas; (iii) for the particular case of fast ignition a $100 \mathrm{~kJ}, 7 \mathrm{PW}, 15$ ps laser pulse is required that is incompatible with the background pressure necessary for ion mitigation. The only realistic ion mitigation strategy to protect the final lenses relies on ion diversion by magnetic or electric fields. Since the distance between the lens and the target is only $8 \mathrm{~m}$, a strong electric field (of the order of a few hundred $\mathrm{kV}$ ) over a few meters is needed for full mitigation. Further work must address the technological viability of such a strategy.

Once we are convinced that ions must be avoided, the next step is to study the effect of X-rays on the final lenses. In the case of the $48 \mathrm{MJ}$ shock ignition target, the X-ray energy is deposited in a very short puls $\mathrm{e}^{23}$ with a width of $\sim 170 \mathrm{ps}$. The energy depth profile is calculated with the appropriate absorption coefficients ${ }^{24}$. The X-rays are rather penetrating (up to the $\mathrm{cm}$ range), however, the energy density deposited in the first $10 \mu \mathrm{m}$ is by far the highest. CODE ASTER calculations carried out under these conditions give the temperature and stress as a function of time for different depths (Figure 5). Both the temperature and stress profiles do not vary along the radial coordinate, $r$, except near the lateral border. One sees that the X-ray radiation leads to a temperature rise of only $14 \mathrm{~K}$ at the sample surface. Assuming that the maximum lens temperature for safe operation is $1200 \mathrm{~K}$, the small temperature rise introduced by the X-rays opens a large operational window to fix the silica base temperature. In next section, it will be discussed the advantage of a base temperature of $800 \mathrm{~K}$ to reduce the accumulation of color centers.

Besides the surface temperature, it is interesting to note that the X-rays lead to the appearance of a temperature gradient parallel to the lens axis. This generates radial stresses due to different expansion of the material at different temperatures (Figure 5). The result is that the hottest region (top surface) suffers compression stress with a maximum of $-0.65 \mathrm{MPa}$.

Let us consider now the situation near the lateral border. We have studied two cases. First, the X-ray irradiation is uniform over the whole inner surface. Second, the lens is held by means of a clamping system that produces shadow on an external ring. Therefore, the inner surface is uniformly irradiated except over the external ring. In the first case, Shear stress appears at the lateral border with a maximum value of $0.31 \mathrm{MPa}$, see Figure 6 (left). In the second case, stress parallel to the lens axis appears underneath the boundary between irradiated and not irradiated surface, see Figure 6 (right). In this case, tensile and compressive stresses appear periodically over the same region. In general, the stresses 
originated by the X-ray radiation are below the safety limits. Further work must be devoted to study fatigue failure mechanisms.

In summary under a scenario similar to HiPER 4a, ions are very detrimental because they lead to melting of the lens. Therefore, ions must be (at least partially) mitigated. On the other hand, X-rays lead to a $14 \mathrm{~K}$ rise of surface temperature and the appearance of stress but in any case much lower than the safety limits. More severe scenarios, as HiPER $4 \mathrm{~b}$, in terms of target yield and operation conditions deserve further work to assess the lifetime of the silica final lenses.
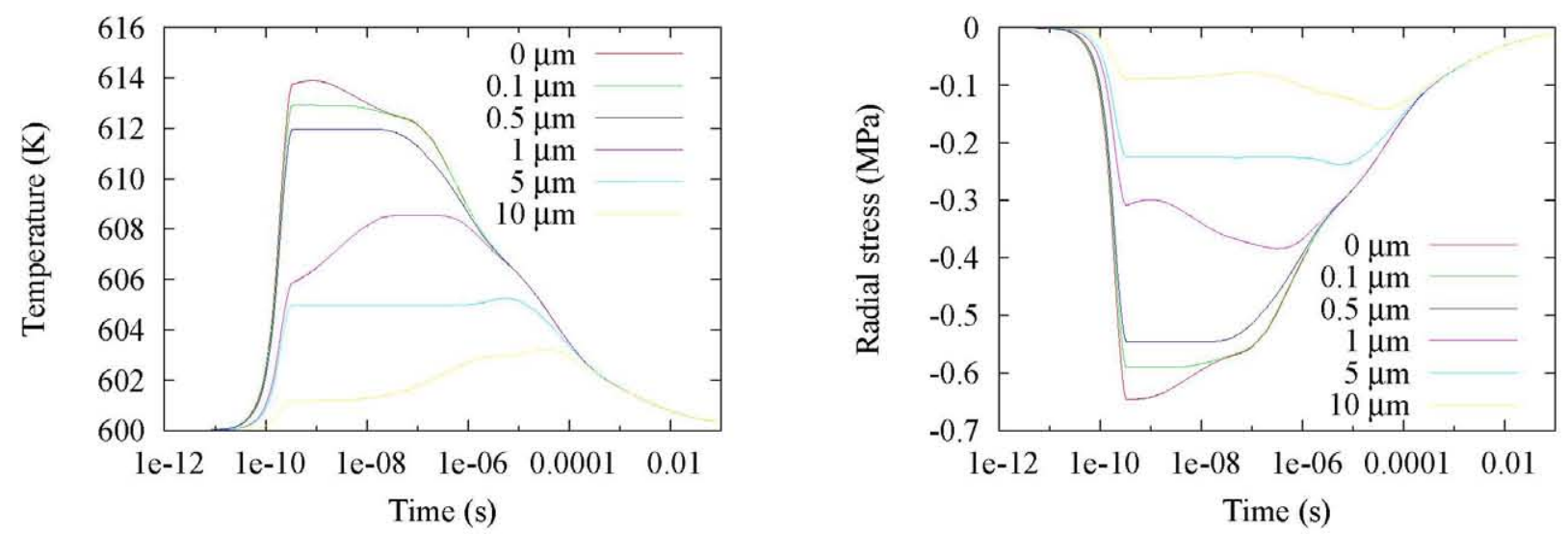

Figure 5. Thermo-mechanical response of the silica final lens subject to one pulse of X-rays generated by the explosion of a $48 \mathrm{MJ}$ shock ignition target (see text). (Left) Temperature as a function of time at different depths. (Right) Radial stress as a function of time at different depths.
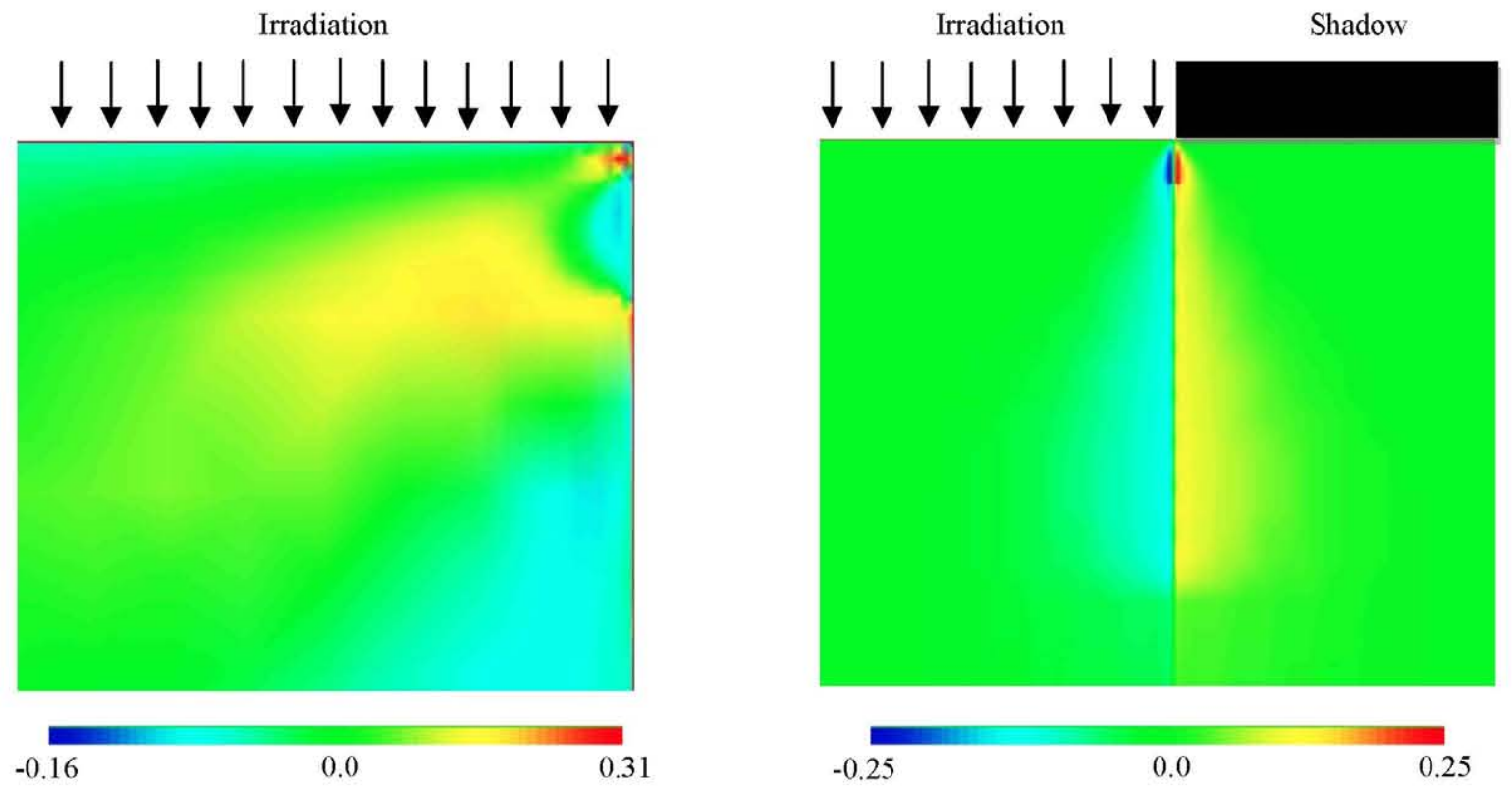

Figure 6. Stress in MPa for different situations. (Left) the whole surface is uniformly irradiated. Shear stress appears at the lateral border. (Right) the whole surface except an outermost ring is irradiated with X-rays. Stress parallel to the lens axis appears underneath the boundary between irradiated and not irradiated surface. 


\section{RADIATION-INDUCED ABSORPTION THROUGH COLOR CENTER FORMATION}

Provided ions are mitigated and once we know that X-ray radiation (48 MJ target) is not deleterious, we need to go deeper into the atomistic effects resulting from combined neutron and gamma irradiation. As discussed in the introduction we have applied a simple model ${ }^{10,11}$ to account for color center formation and evolution with the realistic radiation fluxes obtained in Section 3 for the $48 \mathrm{MJ}$ shock ignition target.

Next, we summarize the major aspects of the model: (i) neutron collision with the silica network produces an oxygen defect center, ODC, (absorption peak at $248 \mathrm{~nm}$ ); (ii) the ODC can be radiolytically converted into an E' center (absorption peak at $214 \mathrm{~nm}$ ) by interaction with $\gamma$-rays; (iii) both ODC and E'centers can be thermally annealed out. These processes are defined by the following equations

$$
\begin{gathered}
\frac{d N_{O D C}}{d t}=\frac{M_{n} \varphi_{n}}{\lambda_{n}}-\beta_{E} D_{\gamma^{\prime}}^{\prime} N_{O D C}-\frac{N_{O D C}}{\tau_{\text {anneal }}}, \\
\frac{d N_{E^{\prime}}}{d t}=\beta_{E} D_{\gamma}^{\prime} N_{O D C}-\frac{N_{E^{\prime}}}{\tau_{\text {anneal }}},
\end{gathered}
$$

where

- $\quad N_{i}$ represent the concentration of the defect center $i$;

- $\quad \beta_{E}$ accounts for the conversion of ODCs into E' centers;

- $\quad \lambda_{n}$ represents the neutron mean free path, see below;

- $\quad M_{n}$ represents the number of defects formed following a primary knock-on atom (PKA), see below;

- $\tau_{\text {anneal }}$ represents the characteristic time for annealing, see below;

- $\varphi_{n}$ represents the neutron flux $\left[\mathrm{cm}^{-2} \mathrm{~s}^{-1}\right]$ on the final lens, see Section 3;

- $\quad D_{\gamma}^{\prime}$ represents the gamma dose rate $[\mathrm{Gy} / \mathrm{s}]$, see Section 3.

In order to get a good estimate of the neutron mean free path $\lambda_{n}$, corresponding to neutrons reaching the sample with energy $E_{n}$, we have run MCNPX for every energy $E_{n}$ using as source term only neutrons of energy $E_{n}$. Figure 7 shows the resulting values of the neutron mean free path for neutrons of different energies.

The number of defects formed following a PKA is given by ${ }^{11}$

$$
M_{n}=0.37 \frac{\text { defects }}{\text { kev - recoil }} \times E_{P K A}(\mathrm{keV}),
$$

where $E_{P K A}$ is the average energy of the neutron-produced PKA and it is related to the neutron energy, $E_{n}$, by ${ }^{11}$

$$
E_{P K A}=\frac{2}{M+2 / 3} E_{n}
$$

where $M=20$ represents the average atomic mass of $\mathrm{SiO}_{2}$. The number of defects formed following a PKA is plotted as a function of neutron energy in Figure 7.

The characteristic time, $\tau_{\text {anneal, }}$, can be written ${ }^{11}$ as

$$
\tau_{\text {anneal }}=\tau_{0} \exp \left(T_{\text {anneal }} / T\right),
$$

being $\tau_{0}$ and $T_{\text {anneal }}$ constants (Table 2).

Finally, the macroscopically accessible absorption coefficient $\alpha$ is related to the obtained defect concentrations by means of the following equation ${ }^{10}$ 


$$
\alpha(\lambda)=\sum_{i} \sigma_{i} N_{i} L_{i}(\lambda) \text { with } L_{i}(\lambda)=\frac{1}{1+\frac{\left(\lambda_{i}-\lambda\right)^{2}}{\left(\Delta \lambda_{i}\right)^{2}}}
$$

where the subscript $i$ denotes a defect center, $\sigma_{i}$ the defect center cross section, $N_{i}$ de defect center density, $\lambda$ the wavelength, $\lambda_{i}$ the peak wavelength for the defect center $i$ and $\Delta \lambda_{i}$ the half width at half maximum for the center $i$. The values we used for the model equations are listed in Table 2.

Table 2. Values used for the parameters of the model.

\begin{tabular}{c|c|c||c|c|c||c|c|c} 
Parameter & Value & Ref. & Parameter & Value & Ref. & Parameter & Value & Ref. \\
\hline$\sigma_{O D C}\left(\mathrm{~cm}^{2}\right)$ & $1.7 \times 10^{-17}$ & 11 & $\sigma_{E^{\prime}}\left(\mathrm{cm}^{2}\right)$ & $3.2 \times 10^{-17}$ & 11 & $\beta_{E}\left(\mathrm{~Gy}^{-1}\right)$ & $7 \times 10^{-5}$ & 11 \\
$\lambda_{O D C}(\mathrm{~nm})$ & 248 & 25 & $\lambda_{E^{\prime}}(\mathrm{nm})$ & 214 & 25 & $\tau_{0}(\mathrm{~s})$ & $4 \times 10^{-10}$ & 11 \\
$\Delta \lambda_{O D C}(\mathrm{~nm})$ & 13 & 25 & $\Delta \lambda_{E^{\prime}},(\mathrm{nm})$ & 15 & 25 & $T_{\text {amneal }}(\mathrm{K})$ & 21000 & 11
\end{tabular}

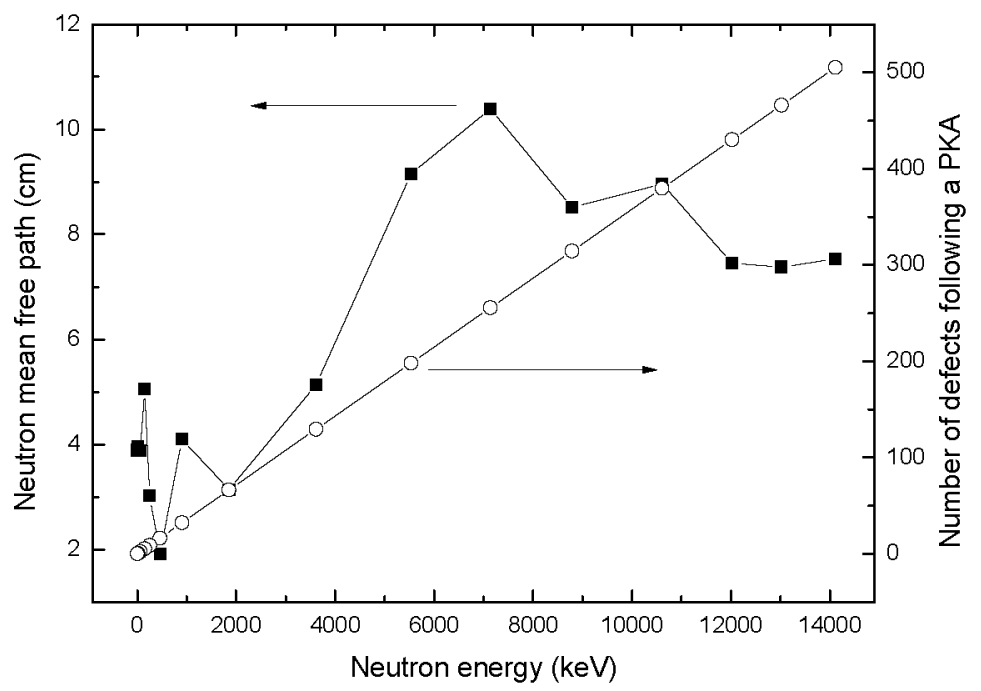

Figure 7. (Left axis) neutron mean free path $\left(\lambda_{n}\right)$ and (right axis) defects following a PKA $\left(M_{n}\right)$ as a function of neutron energy.
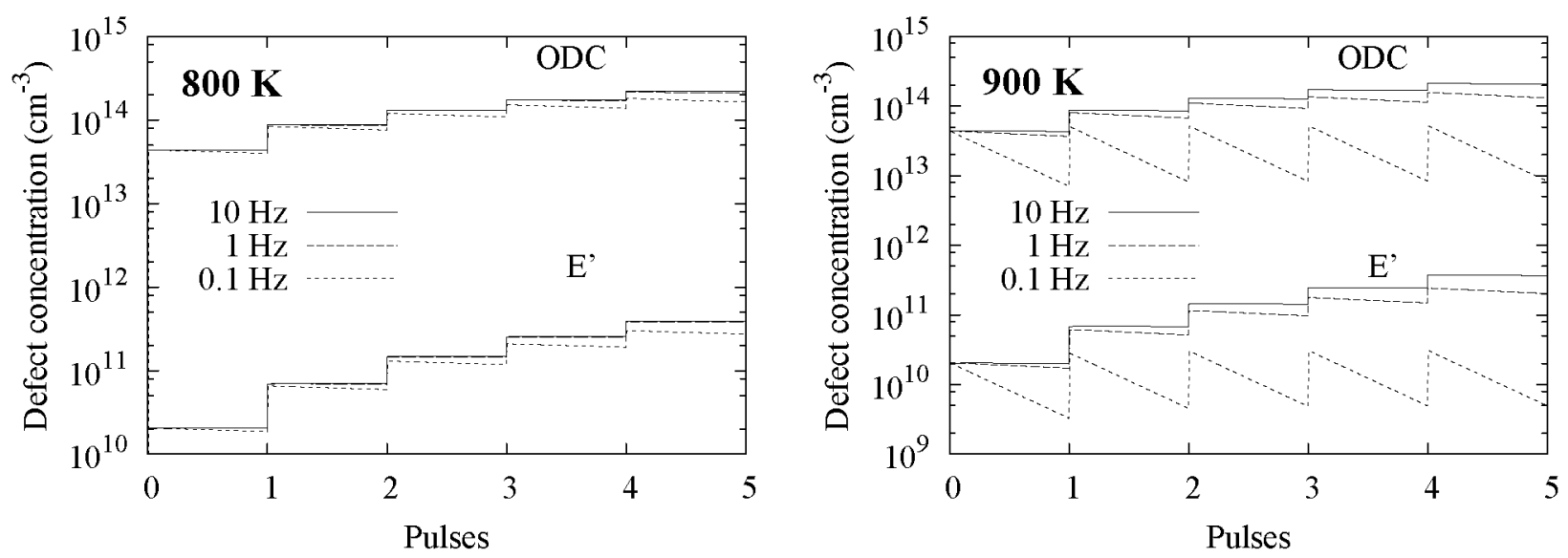

Figure 8. (Left) calculations of ODC and E' concentration accumulated in the final lens as a function of the number of $48 \mathrm{MJ}$ target explosions for different explosion rates and sample temperature at $800 \mathrm{~K}$. (Right) same calculations for sample temperature at $900 \mathrm{~K}$. 
We have applied this model to a silica sample (lens) located $8 \mathrm{~m}$ away from a 48 MJ shock ignition target (HiPER 4a geometry, Section 2) and subject to simultaneous neutron and $\gamma$-fluxes (calculated in Section 3). We ignore the effect of $\mathrm{X}$-rays resulting from the explosion and assume that ions are mitigated to avoid the melting of the lens (Section 4).

Figure 8 shows the ODC and E' concentration accumulated in samples at different temperatures after every explosion for three different explosion rates $0.1,1$ and $10 \mathrm{~Hz}$. The resulting ODC concentration is three orders of magnitude higher than the E' concentration. This reveals that the $\gamma$-dose rate is too low to promote effective ODC conversion to E' centers according to equation (2) of the model. In addition, we observe that the higher the explosion rate the higher the defect accumulation. This is logical because a higher rate implies a shorter time interval between explosions and thus a reduced defect annealing. The temperature effect is clearly observable when comparing both panels: the higher the temperature, the higher the number of annealed defects. This is especially remarkable at low explosion rates $(0.1 \mathrm{~Hz})$ that makes possible significant reduction of the defect concentration during the time interval between explosions. It is evident that defect annealing is not very important at low temperatures. In fact, at room temperature it has no effect for time scales comparable to the explosion period (inverse of explosion rate).
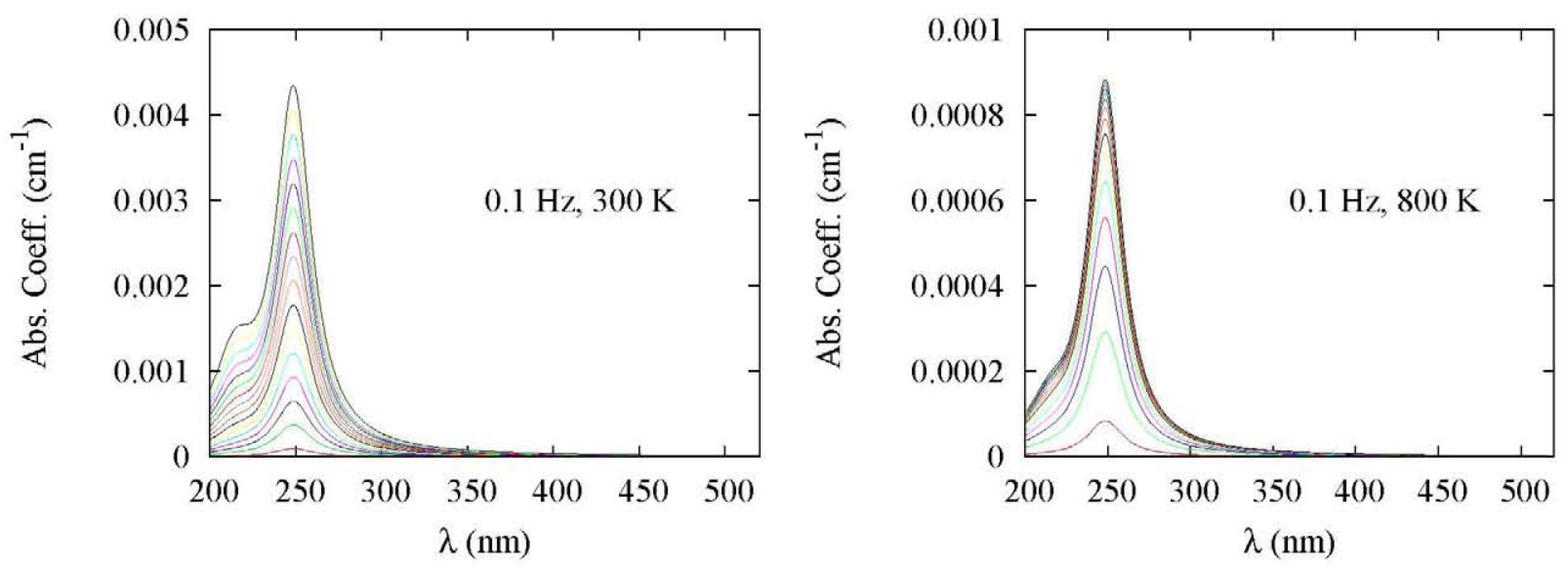

Figure 9. (Left) ODC and E' contribution to the absorption coefficient, $\alpha$, for a sample kept at $300 \mathrm{~K}$ during exposure to $48 \mathrm{MJ}$ target explosions at a rate of $0.1 \mathrm{~Hz}$. (Right) the same calculations for sample temperature at $800 \mathrm{~K}$. Curves given every 3 pulses ( $30 \mathrm{~s}$ ). The lowest curves correspond to 3 pulses and the highest to 48 . Note that the vertical axes of the plots are not at the same scale.

From equation (6) we can relate the defect concentrations to the absorption coefficients. Figure 9 shows the ODC and E' contribution to the absorption coefficient for samples at two temperatures $(300$ and $800 \mathrm{~K})$ and low explosion rate $(0.1$ $\mathrm{Hz}$ ). The annealing effect is very evident here. Samples subject to irradiation at $300 \mathrm{~K}$ show a much higher absorption coefficient than those at $800 \mathrm{~K}$ (note that the scales are different). In addition, one can clearly distinguish both the ODC peak $(248 \mathrm{~nm})$ and the E' peak $(214 \mathrm{~nm})$ for the sample at $300 \mathrm{~K}$. We can also distinguish both peaks in the case of the sample at $800 \mathrm{~K}$, however, the E' contribution is less marked (small shoulder) in this case due to significant annealing.

We have computed the rise in the absorption coefficient, $\alpha$, due to the accumulation of ODC and E' centers. In order to make predictions about silica behavior for final lenses of HiPER 4a, we calculate the optical absorption, $A$, by means of

$$
A=1-\exp [-\alpha(\lambda) d] \text {, }
$$

where $d$ is the thickness of the silica sample. Figure 10 shows some results concerning absorption at $350 \mathrm{~nm}$ due to ODC and E' center accumulation with an explosion rate of $0.1 \mathrm{~Hz}$. Regardless the sample temperature the optical absorption remains low. At room temperature, the optical absorption reaches $5 \%$ in about 6000 pulses (not shown). This is comparable to the number of shots planned in HiPER 4a. Therefore, if we take $5 \%$ as the upper limit for the optical absorption, according to our calculations, the lifetime of silica final lenses is comparable to the facility lifetime. Anyway, the facility is designed to permit replacement of the lenses by means of remote handling systems. 
The calculations show that there exists an operational window that would cover more pessimistic scenarios for HiPER 4a and even much more exigent scenarios as HiPER $4 \mathrm{~b}$. Going back to Figure 10 one realizes that the absorption curves reach very rapidly ( 50 pulses) a saturation value at temperatures above $\sim 800 \mathrm{~K}$ because defect annealing effectively competes with defect creation. This temperature is lower than the expected reactor wall base temperature. Therefore, one can argue that under reactor condition (HiPER $4 \mathrm{~b}$ ) it will be in principle possible to maintain the lenses at a temperature high enough to promote effective annealing of defects preserving good optical transmission. For comparison we show in the right panel (Figure 10) the optical absorption of lenses with different thickness. As expected, we obtain that the thinner the lenses the lower the absorption. Therefore, another possible way to keep the optical absorption low is to use thinner components.

Finally, we consider thermal annealing as a method to heal the lenses. Let us consider a $5 \mathrm{~cm}$-thick lens and assume that its optical absorption at $350 \mathrm{~nm}$ is due to ODC and E' centers. An absorption of $5 \%$ corresponds to $N_{O D C}=2.4 \times 10^{17} \mathrm{~cm}^{-3}$ and $N_{E}=6.4 \times 10^{15} \mathrm{~cm}^{-3}$. Figure 11 shows the effect of thermal annealing at different temperatures to reduce the optical absorption to zero. The main conclusion is that an effective annealing requires temperatures of at least $700 \mathrm{~K}$ to be practical in terms of annealing time. Lower temperatures require too long annealing times to be practical (days at $600 \mathrm{~K}$ ).
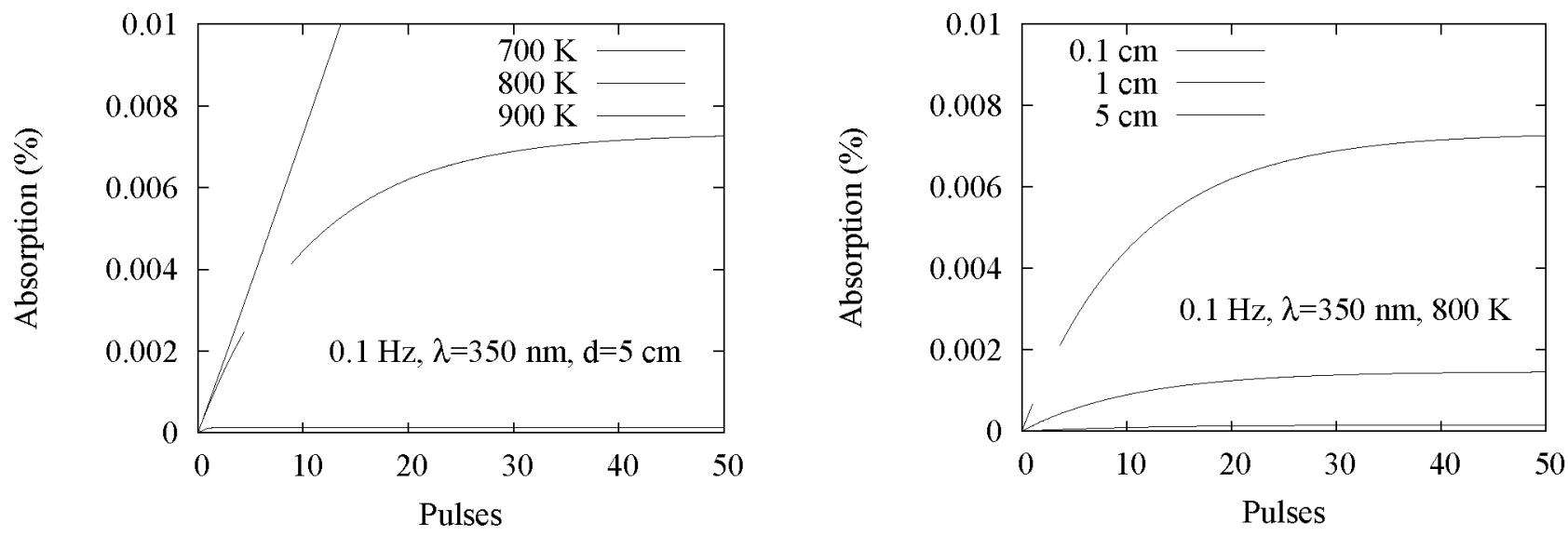

Figure 10. (Left) optical absorption at $\lambda=350 \mathrm{~nm}$ of a $5 \mathrm{~cm}$-thick silica sample kept at different temperatures due to ODC and E' formation as a consequence of periodic $48 \mathrm{MJ}$ target explosions at a rate of $0.1 \mathrm{~Hz}$. (Right) the same for samples of different thickness kept at $800 \mathrm{~K}$.

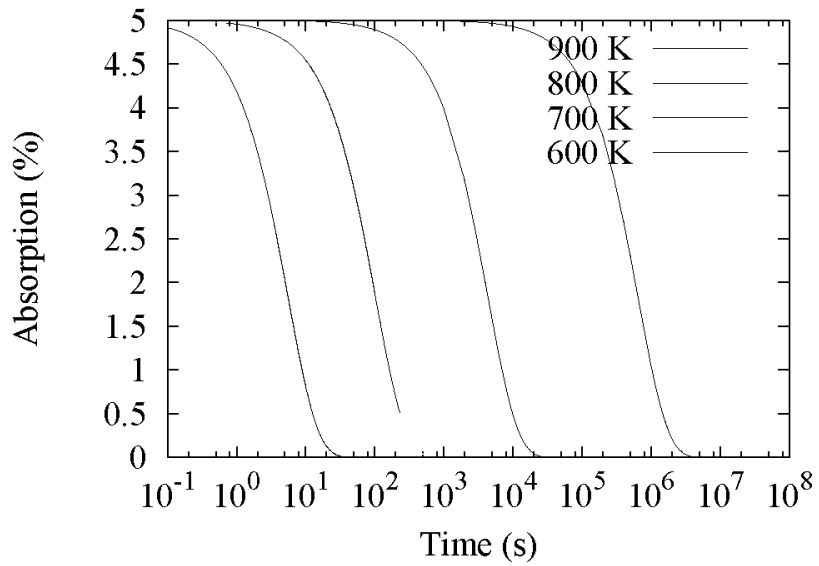

Figure 11. Recovery of optical absorption by means of themal annealing at the indicated temperatures. 


\section{SUMMARY AND FURTHER WORK}

We have investigated the performance of silica lenses located at $8 \mathrm{~m}$ from a $48 \mathrm{MJ}$ shock ignition target, i.e., a realistic situation for the phase 4a of HiPER. We studied the target yield in terms of radiation affecting the lens. As it generally occurs for direct targets, ions represent a major threat for the sample accounting to $\sim 24 \%$ of the target energy, while Xrays account only to $\sim 1 \%$. Ions and X-rays lead to thermo-mechanical effects in the silica lenses. In fact, ions turn out to be so deleterious (lead to sample melting) that must be avoided by (partial or total) mitigation strategies. On the other hand, X-rays produce temperature rise an stresses, none of them are detrimental.

Next, we calculated the neutron flux and $\gamma$-dose rate reaching the lens after every explosion. The simultaneous neutron and gamma irradiation leads to the appearance of color centers. We have used a simple model to study the evolution of oxygen deficient centers (ODC) and E' centers under the $48 \mathrm{MJ}$ explosions for different sample temperature and thickness. The major conclusion is that the lifetime of silica final lenses operating at room temperature is comparable to the expected lifetime of the HiPER 4a facility. Sample temperatures above $\sim 800 \mathrm{~K}$ lead to a saturation of the optical absorption, which would make possible to operate in reactor mode (continuous shooting). This temperature seems not so high for a reactor in operation. If it is not possible to keep that temperature during operation or if the irradiation conditions are much more severe one could go to thinner lenses or carry out thermal annealing treatments to refresh the lenses.

Despite the huge experimental effort carried out to irradiate samples under realistic irradiation conditions, it has been difficult to mimic laser fusion irradiation conditions, (high neutron flux pulses of $14 \mathrm{MeV}$ neutrons simultaneously to gamma irradiation). Validation of our calculations will require to carry out experiments under such conditions. Thanks to the National Ignition Facility (NIF), recently in operation, these experiments seem feasible in a near future.

Experiments at NIF can be done in a single shot basis. Therefore, silica material qualification to reactor conditions as those of HiPER $4 \mathrm{~b}$ will not be straightforward. Experiments done with a few pulses will have to be extrapolated to conditions of about 1 million pulses per day. This means that further work on radiation-matter interaction is still needed. One important point here relies on the fact that color center formation takes place by purely ionizing radiation $(\gamma$-rays, electrons), i.e., it is of paramount importance to understand the physical bases of color center formation in regions of materials with very dense electronic excitation. Contrary to defect formation by particle collision, electronic excitation mechanisms are not well understood. We are working to include electronic excitation effects in Molecular Dynamics (MD) simulations and we expect to provide a better picture of defect evolution and its influence on the macroscopic optical properties.

\section{REFERENCES}

[1] J.L. Bougarde et al., "Diagnostic components in harsh radiation environments: Possible overlap in R\&D requirements of inertial confinement and magnetic fusion systems," Rev. Sci. Instrum. 79, 10F304 (2008).

[2] K. Yu. Vukolov, "Radiation effects in window materials for ITER diagnostics," Fusion Engineering and Design 84,1961 (2009).

[3] L. Skuja, M. Hirano, H. Hosono and K. Kajihara, "Defects in oxide glasses," Phys. Stat. Sol. (C) 2, 15 (2005).

[4] W. Primak, L.H. Fuchs, P. Day, Phys. Rev. 92, 1064 (1953).

[5] E.J. Friebele, Phys. Rev. Lett. 42, 1346 (1979).

[6] M. León, P. Martin, D. Bravo, F.J. López, A. Ibarra, A. Rascón, F. Mota, J. Nucl. Mater. 374, 386 (2008).

[7] D.L. Griscom, M.E. Gingerich, E.J. Friebele, Phys. Rev. Lett. 71, 1019 (1993).

[8] H. Imai, K. Arai, J. Isoya, H. Hosono, Y. Abe, H. Imagawa, Phys. Rev. B 48, 3116 (1993).

[9] H. Hosono, M. Mizuguchi, H. Kawazoe, T. Ogawa, Appl. Phys. Lett 74, 2755 (1999).

[10] C. D. Marshall, J. A. Speth, S. A. Payne, "Induced optical absorption in gamma, neutron and ultraviolet irradiated fused quartz and silica," J. Non-Cryst. Solids, 212, 59 (1997).

[11] J.F. Latkowski, A. Kubota, M.J. Caturla, S.N. Dixit, J.A. Speth, S.A. Payne, "Fused silica final optics for inertial fusion energy: radiation studies and system-level analysis," Fusion Science and Technology 43, 540 (2003).

[12] D. L. Griscom, "Optical properties and structure of defects in silica glass," J. Ceram. Soc. Jpn., 99, 923 (1991). 
[13] R. A. Weeks, "The many varieties of E' centers: A review," J. Non-Cryst. Solids, 179, 1 (1994).

[14] V V Afanas'ev, A Stesmans, "Charge state of paramagnetic E' centre in thermal $\mathrm{SiO}_{2}$ layers on silicon," J. Phys.: Condens. Matter. 12, 2285 (2000).

[15] G. Zimmerman, D. Kershaw, D. Bailey, J. Harte. "LASNEX code for inertial confinement fusion", J. Opt. Soc. Am. 68, 549 (1978)

[16] J. Perkins. Internal report.

[17] M.S. Tillack and J. E. Pulsifer: "Long-term survival of grazing-incidence metal mirrors for laser fusion", Fusion Science and technology 56, 446-451 (2009).

[18] D.B. Pelowitz, 2005 MCNPX User's Manual and extensions, LA-CP-05-0369.

[19] Wu Y, FDS Team 2009 Fusion Engineering and Design 84, 1987-92.

[20] http://www.code-aster.org/

[21] J.F. Ziegler, J.P. Biersack, U. Littmark, The Stopping and Ranges of Ions in Solids, Pergamon Press, New York, 1985, see also the SRIM webpage, http://www.srim.org/.

[22] R.W. Petzoldt et al., "Direct drive target survival during injection in an inertial fusion energy power plant," Nucl. Fusion 42, 1351 (2002).

[23] T.A. Heltemes, D.R. Boris, G.A. Moses, M. Fatenejad, "Simulation of thermal response and ion deposition in the HAPL target chamber $1 \mathrm{~mm}$ tungsten armor layer using the improved BUCKY code", Fusion Engineering and Design 82, (2007) 175-187

[24] http://physics.nist.gov/PhysRefData/XrayMassCoef/ElemTab/z74.html

[25] N. Kuzuu, "X-ray induced absorption bands in synthetic fused silicas: OR content dependence of intensities of X-ray-induced absorption bands in type-III fused silicas", Journal of Non-Crystalline Solids 179 (1994) 170-178 